\title{
Electronic Structure of Tetracyanonickelate(II)
}

\author{
Julius J. Oppenheim*†, Brendon J. McNicholas†, Jayce Miller†, Harry B. Gray†
}

$\dagger$ Division of Chemistry and Chemical Engineering, California Institute of Technology, Pasadena, California 91125 , United States 
Example TD-DFT Calculation Input Parameters:

! PBE0

! ma-def2-TZVPP TIGHTSCF Opt Grid3 FinalGrid5 xyzfile

! CPCM

$\%$ cpcm

epsilon 37.5

end

* xyzfile -21 guess.xyz

\$new_job

! ma-def2-TZVPP Grid5 FinalGrid6 TightSCF

! PBE0

! CPCM

$\%$ cpcm

epsilon 37.5

end

$\%$ tddft

nroots 25

maxdim 5

tda true

triplets true

end

*xyzfile -21

Example of EOM-CCSD calculation

! RHF EOM-CCSD def2-SVP TightSCF

\%maxcore 8000

!moread

\%moinp "16eeeom.gbw"

$\%$ mdci

NInitS 30

nroots 9

DoCOSXEOM true

DoAOX3e true

KCOpt KC_AOBLAS

end 


$$
\begin{aligned}
& \text { * xyz }-21 \\
& \text { Ni } 000 \\
& \text { C } 1.8700 \\
& \text { C }-1.8700 \\
& \text { C } 01.870 \\
& \text { C } 0-1.870 \\
& \text { N } 3.0300 \\
& \mathrm{~N}-3.0300 \\
& \text { N } 03.030 \\
& \text { N } 0-3.030
\end{aligned}
$$

Example of CASSCF calculation

! CASSCF ma-def2-TZVPP NoIter

\%maxcore 600000

!moread

\%moinp "11.gbw"

! CPCM

$\%$ cpcm

epsilon 37.5

end

\%casscf

nel 10

norb 10

mult 3,1

nroots 4,5

NEVPT2 SC

nevpt

d4tpre 1e-8

end

end

$*$ xyz -21

Ni 000

$\mathrm{C} 1.8700$

$\mathrm{C}-1.8700$

C 01.870

C $0-1.870$

N 3.0300

$\mathrm{N}-3.0300$ 
$\mathrm{N} 03.030$

$\mathrm{N} 0-3.030$

* 